\title{
2020 Physics Nobel laureate Roger Penrose and the Penrose pattern as a forerunner of generalized crystallography
}

\author{
Istvan Hargittai ${ }^{1} \cdot$ Balazs Hargittai $^{2}$ \\ Accepted: 20 October 2020 / Published online: 31 October 2020 \\ (C) Springer Science+Business Media, LLC, part of Springer Nature 2020
}

\section{Nobel recognition}

Three physicists shared the Nobel Prize in Physics for 2020. Roger Penrose (1931, Fig. 1) received half of the prize "for the discovery that black hole formation is a robust prediction of the general theory of relativity." The other half was divided between Reinhard Genzel (1952) and Andrea Ghez (1965) "for the discovery of a supermassive compact object at the center of our galaxy." Penrose's discovery was the result of mathematical research into the general theory of relativity, whereas Genzel and Ghez utilized the most up-to-date technological advances in astronomy to make their observations. Some of the formulations of the Penrose discovery read as if the black hole had predicted the general theory of relativity, and some others, as if the formation of black holes was a consequence of the theory. In any case, Penrose uncovered the relationship between the black holes and the general theory of relativity. The black holes are super heavy formations and they swallow everything; even light cannot escape from them. Albert Einstein did not consider the kind of relationship Penrose discovered and did not even believe in the existence of black holes. Penrose discussed the nature of the black holes within a decade following Einstein's death. Penrose described the singularity occurring in the depth of black holes where the known laws of nature lose their validity. We don't know that kind of laws may apply at that point. Penrose discussed the

Dedicated to Alan L. Mackay for his 95th birthday

Istvan Hargittai

istvan.hargittai@gmail.com

Balazs Hargittai

bhargittai@francis.edu

1 Budapest University of Technology and Economics, Budapest 1111, Hungary

2 Saint Francis University, Loretto, PA 15940, USA

details of his discovery both in research papers and in bestselling semi-popular books [1-5].

Alfred Nobel initiated his prizes to award great discoverers, not just great scientists. The two do not always coincide although at the early stages of the prize most awardees were both great discoverers and great scientists. Nowadays, it does not happen so often that the awardees are not only great discoverers but also great scientists. Roger Penrose is both and this makes even a cursory acquaintance with his oeuvre and personality especially rewarding.

\section{Background}

We recorded a long conversation with Roger Penrose twenty years ago, in 2000, at Oxford University, and we published it in 2005, in the fifth volume of our Candid Science book series [6]. In each of the six-volume Candid Science book series, there were at least 36 conversations of which at least 18 were with Nobel laureates [7]. In the fifth volume, there were 19 Nobel laureates when the book appeared in 2005, and there are 21 today. One of the two additions was Dan Shechtman (conversation in 1995) who received the Nobel Prize in 2011 for the discovery of quasicrystals. The other addition is Roger Penrose.

There was no interaction between Shechtman and Penrose, except for a chance meeting, and their Nobel distinctions were awarded for discoveries in faraway domains of science. However, their interests strongly overlapped in the area of the symmetry features of extended structures. This will be in the focus of the next segment, but first a little more about Roger Penrose.

Roger Penrose was born in Colchester, Essex, in Eastern England. The Penroses were a well-known intellectual family. His father, Lionel Penrose (1898-1972), was interested in genetics and the inheritance of mental illness. He considered Francis Galton an important scientist, but opposed eugenics. When Lionel Penrose was appointed to the Galton Chair of 
Fig. 1 Roger Penrose in 2000 in Oxford (photo by Istvan Hargittai)

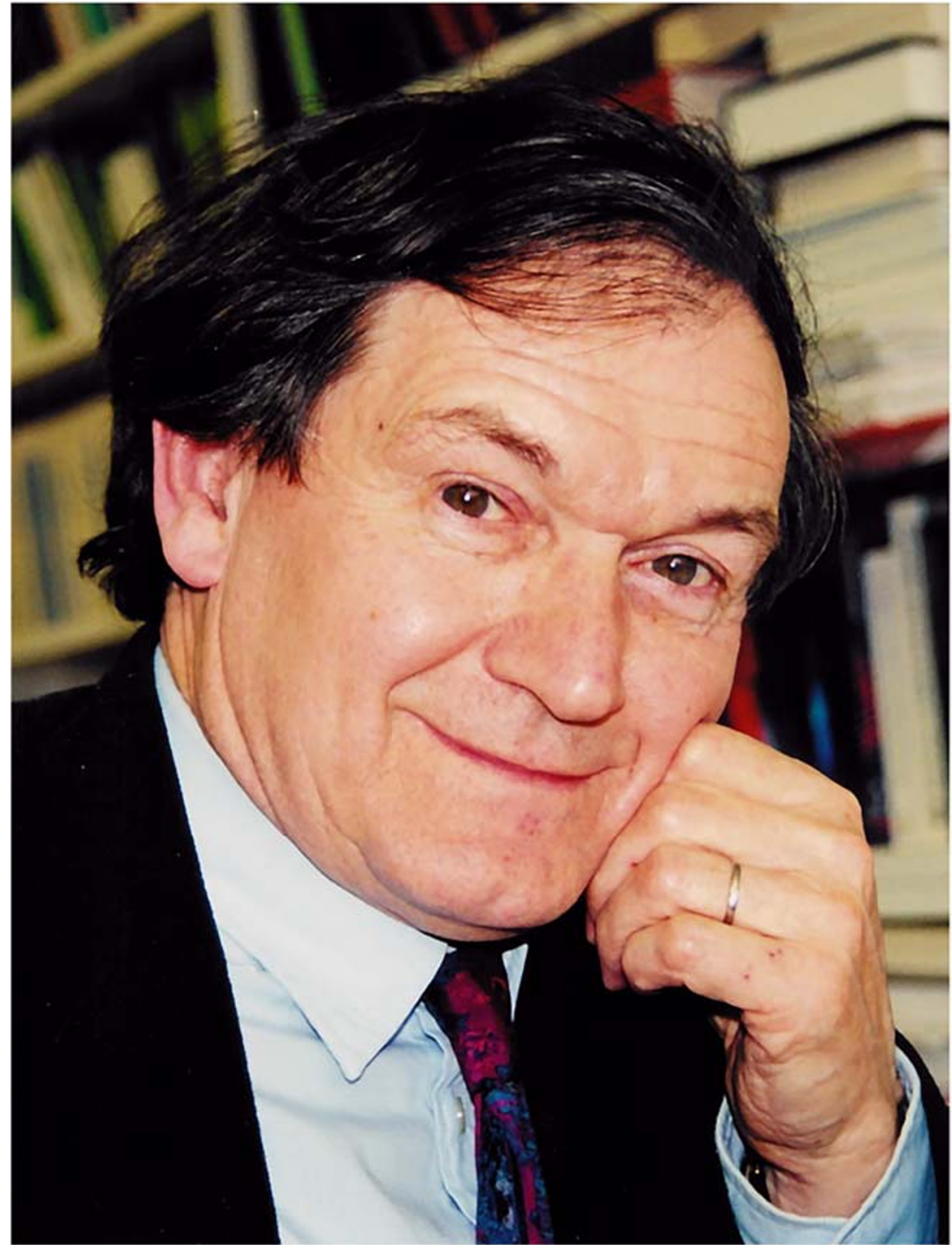

Eugenics at University College London, he wanted to change the name of the chair from the start, and it was eventually renamed to Galton Chair of Human Genetics. Also, he managed to change the name of the journal Eugenics to Human Genetics. He loved science and he loved mathematical puzzles and similar entertainment and he drew no sharp line between serious science and fun. Neither does Roger, who was very close to his father. They used to take long walks together. They looked at the plants and were amazed by the scattered leaf arrangements around the stem, recognizing the underlying Fibonacci numbers in them. Roger has three siblings, one is a geneticist, another is a physicist, and one is a psychologist and a ten times British chess champion. Chess was a big deal in the family; the father solved chess puzzles and constructed others; but Roger was not interested in chess.

Roger liked to doodle, especially during boring meetings. Many people do this, but for Roger, it was often connected to solving mathematical puzzles. Both Lionel and Roger liked
MC Escher's unusual graphics and Roger even visited the Dutch artist. He shared some of his own and his father's drawings of mathematical puzzles with the artist who then developed his graphics from them. Some have become well known, but most people are not aware of the origin of those drawings.

Beyond his father's influence, mentors and professors at Cambridge University helped Roger's development. Roger was initially dedicated to pure mathematics. Dennis Sciama (1926-1999) inculcated in him an intense interest in physics and enhanced his knowledge of physics. They became friends and used to go to Stratford together to watch Shakespeare plays. They talked about physics during the car rides. In Cambridge, Roger attended many courses regardless of whether or not they were directly connected with his principal studies. He attended Paul Dirac's quantum mechanics, Hermann Bondy's course on the theory of relativity, and S.W.P. Steen's mathematical logic. In Steen's course, he learned about Turing machines and Gödel's theorem. Roger 
read everything Schrödinger had written on the semi-popular level. Penrose spent his post-doctoral studies at Princeton where he associated mostly with John Arhibald Wheeler. Roger was fascinated by Wheeler's interest to bring much geometry into physics, as he meant to incorporate all of physics into geometry. He benefited from Wheeler's deep knowledge of the theory of relativity, and from getting to know a broad circle of Wheeler's colleagues active in this field. Wheeler liked to say that Roger's stint at Princeton was due to the efforts by NATO to catch up with the Soviet Union.

The debates between Penrose and Stephen Hawking (1942-2018) received great publicity and some compared them even to the Einstein-Bohr debates. Penrose and Hawking wrote papers together on the ideas of singularities. Penrose thought that he influenced Hawking's direction of research in this area. In their joint papers, they used topological arguments to show that gravitational collapse leads to a singular state producing the Big Bang. What people may refer to as their debate was a book in which they communicated their series of lectures, alternating one after the other. This was not a debate though. The disagreement could perhaps be expressed in the way the two considered quantum mechanics. Penrose looked at it as something still evolving, but Hawking could not accept a view about a changing quantum mechanics. This issue occupied Penrose for a long time. He even thought out a large-scale experiment of astronomical enormity, which could be carried out at huge expense.

\section{Collapse of a dogma in crystallography}

Giants in the history of science, such as Albrecht Dürer, Johannes Kepler, and J. Desmond Bernal, were engaged at one time or another in investigating the properties of the pentagon, the pentagonal dodecahedron, and the impossibility of fivefold symmetry in extended structures - at least, this was a powerful dogma in classical crystallography.

Penrose does not believe in any clear line between doodling and serious research. Indeed, what his doodling was initially would eventually lead to the Penrose patterns. These patterns were to prove instrumental in bringing down the dogma of classical crystallography about the impossibility of fivefold symmetry in the world of crystals. This began when he noticed a pentagonal logo in a letterhead of one of his correspondents. There was a pentagon in its center, surrounded by five same-size pentagons making a larger pentagon. The contour of this scheme left five triangles uncovered in this larger pentagon. Penrose was looking for ways to cover these triangles in this larger pentagon. He cut up a seventh same-size pentagon, which yielded the needed triangles, and left a smaller-size pentagon unused. This scheme is illustrated in Fig. 2, resulting in the simplest Penrose pattern. This happened about 1972, and it was still more doodling than serious science.

He thought of it more as a mathematical curiosity and published an article about it in a rather obscure mathematical periodical [8]. The article itself was based on his lecture at a meeting focusing on aesthetics in mathematics. From the start, the question about the possible applications of the Penrose pattern in crystallography arose. Still, the Penrose pattern might have disappeared in oblivion. This did not happen due primarily to two individuals. One was Martin Gardner, a philosopher by training, who edited a column of mathematical curiosities in Scientific American. In contrast to the obscure mathematical journal, it was well known and most popular. Gardner had an excellent ability to recognize what was important and interesting and put the Penrose pattern onto the cover of a 1977 issue. The artistic representation of the cover illustration was prepared by no less a mathematician than John Conway. Gardner's article was based on his discussion with Conway [9]. This article made the Penrose pattern famous.

The British crystallographer, Alan L. Mackay, was the other individual who recognized the extraordinary potentials of Penrose patterns in crystallography. He had a simulated light diffraction pattern produced from a Penrose pattern. He published it in 1982 [10], and this diffraction pattern displayed symmetry forbidden by the rules of classical crystallography. Mackay issued a warning that if we assume the validity of the dogma about forbidden symmetries, we might ignore them even if we would observe them. At about the same time, the Israeli materials engineer of the TECHNION, Dan Shechtman, visiting at the US National Bureau of Standards (as it was then), did observe forbidden symmetry in the electron diffraction pattern of an aluminum/manganese alloy. Although he was not familiar with Mackay's warning, he did not ignore it just because the classical dogma had taught him to do so. He documented his observation meticulously in 1982, but could publish it only in 1984 [11]. Shechtman immediately recognized the extraordinary significance of his observation, but it took much effort to convince others about its validity [12].

Crystals, according to classical crystallography, have regular and periodic structures. Amorphous materials are nonregular and non-periodic. The Penrose patterns implied and Mackay's simulation experiments suggested that there could exist extended structures that were regular, but non-periodic. Today, these structures, called quasicrystals, belong to the domain of crystallography, meaning that the definition of crystals has expanded. This was a significant step for crystallography to become-using Mackay's terminologygeneralized crystallography, a more general science of structures. This development was due primarily to the activities and discoveries of Penrose, Mackay, and Shechtman. The contribution of the theoretical physicists Paul Steinhardt and Dov Levine is also to be mentioned. They coined the term 

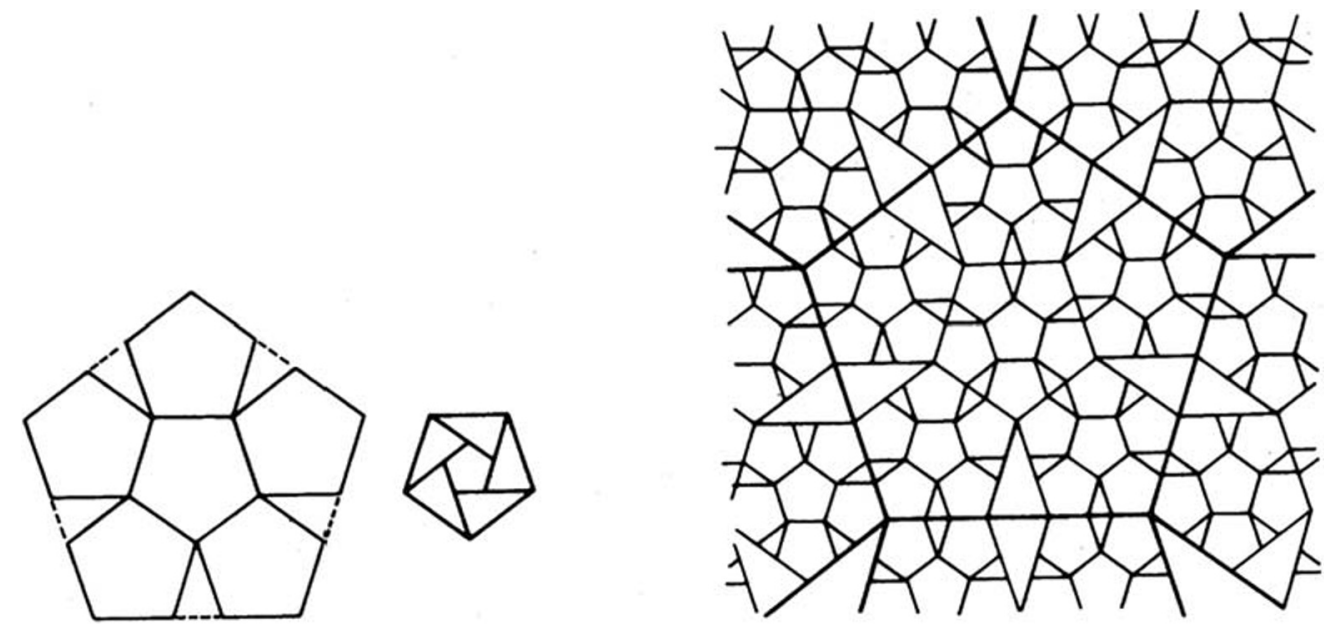

Fig. 2 Developing the simplest Penrose pattern of regular pentagons with changing sizes; courtesy of Alan L. Mackay

quasicrystal and had worked out a theoretical model to describe regular and non-periodic structures. Alas, it appears that the dogma of classical crystallography forbidding fivefold symmetry was overwhelming for them, and they kept their model in their drawer. They published it only in the wake of the publication of Shechtman's observation [13]. This only emphasizes Mackay's courage and integrity. ${ }^{1}$ The discovery brought about a paradigm change in broader domain of science than just crystallography - in chemistry, physics, and materials science. It has appeared in artistic creations as well.

Looking back to the origin of Penrose's interest in covering the surface with regular pentagonal shapes, he himself was keen to understand it. He was not aware initially of Kepler's attempts, but had seen Dürer's picture at some stage; it did not pique his interest though. Then, Penrose saw Kepler's drawings in a book [14] and they touched him, perhaps even psychologically. This still did not prompt Penrose to action, but he developed a friendly attitude towards them. Gradually, he became curious as to what one might do with pentagons in terms of tiling. When he produced what became known as the Penrose patterns, he found them quite close to Kepler's attempts.

Johannes Kepler published a small book in 1611 , De nive hexangula [15]. Kepler admired the shape and symmetry of the six-cornered snowflakes. The book was a milestone in the history of science because for the first time it was enunciated that the external shape of crystals forms as a consequence of internal structure. Today it is obvious that the hexagonal shape

\footnotetext{
${ }^{1}$ When Shechtman's Nobel Prize for the quasicrystal discovery was announced in October 2011, Penrose wrote a letter to Mackay in which he stressed: "If anyone had been clear in the prediction that quasi-symmetric 5fold/10-fold patterns might underlie a completely new area of crystallography - where the very way that such materials might indeed be identified through their characteristic diffraction patterns - it was clearly you." (Private communication from Robert H. Mackay to the authors, by email on October 9, 2020.)
}

and symmetry of the beautiful snowflakes is a consequence of the hexagonal three-dimensional arrangements of the water molecules in the snow crystal. In 1975, Alan Mackay and Roger Penrose met, and Mackay informed Penrose about the simulated light diffraction experiment of the Penrose pattern. At the time, Penrose was experimenting with an extended pentagonal network that could be considered to be a pentagonal snow crystal. Mackay's son, Robert, was also present and Penrose gave him a copy of his hand-drawn pattern. Robert was a student at York at the time and when he returned to his computer, he automated Penrose's drawing. The computer ran out of time at some point hence the snow crystal pattern remained incomplete. This incomplete pattern had the advantage of showing many different parts of its generation, and it is more informative about how it came about than a complete pattern would have been. This computer generated pattern is shown in Fig. 3. Penrose and the Mackays thought this to be a theoretical exercise and years later they were astonished when Shechtman turned up the real thing. Thirty years later, Robert asked Penrose to autograph the drawing (Fig. 3).

It was an interesting process from the doodling by Penrose to his pattern, known as Penrose pattern. The best known among them is depicted in Fig. 4. It is a good example of how a discovery may happen when there is no such aim initially. The discovery of quasicrystals could have developed in the Penrose-Mackay-Shechtman succession, but this remains a thought experiment. An experimental scientist, like Shechtman, could have embarked on a search of what had become known as quasicrystals. This is not how it happened though. Shechtman was not aware of the Penrose pattern, neither of the Mackay simulated experiment nor his warning. That he did not brush off the "forbidden" symmetry in the electron diffraction pattern when it appeared on his screen, was due to his researcher's acumen. That he stood by it for years in spite of criticism, even ridicule, was due to his stubbornness and perseverance [16]. 


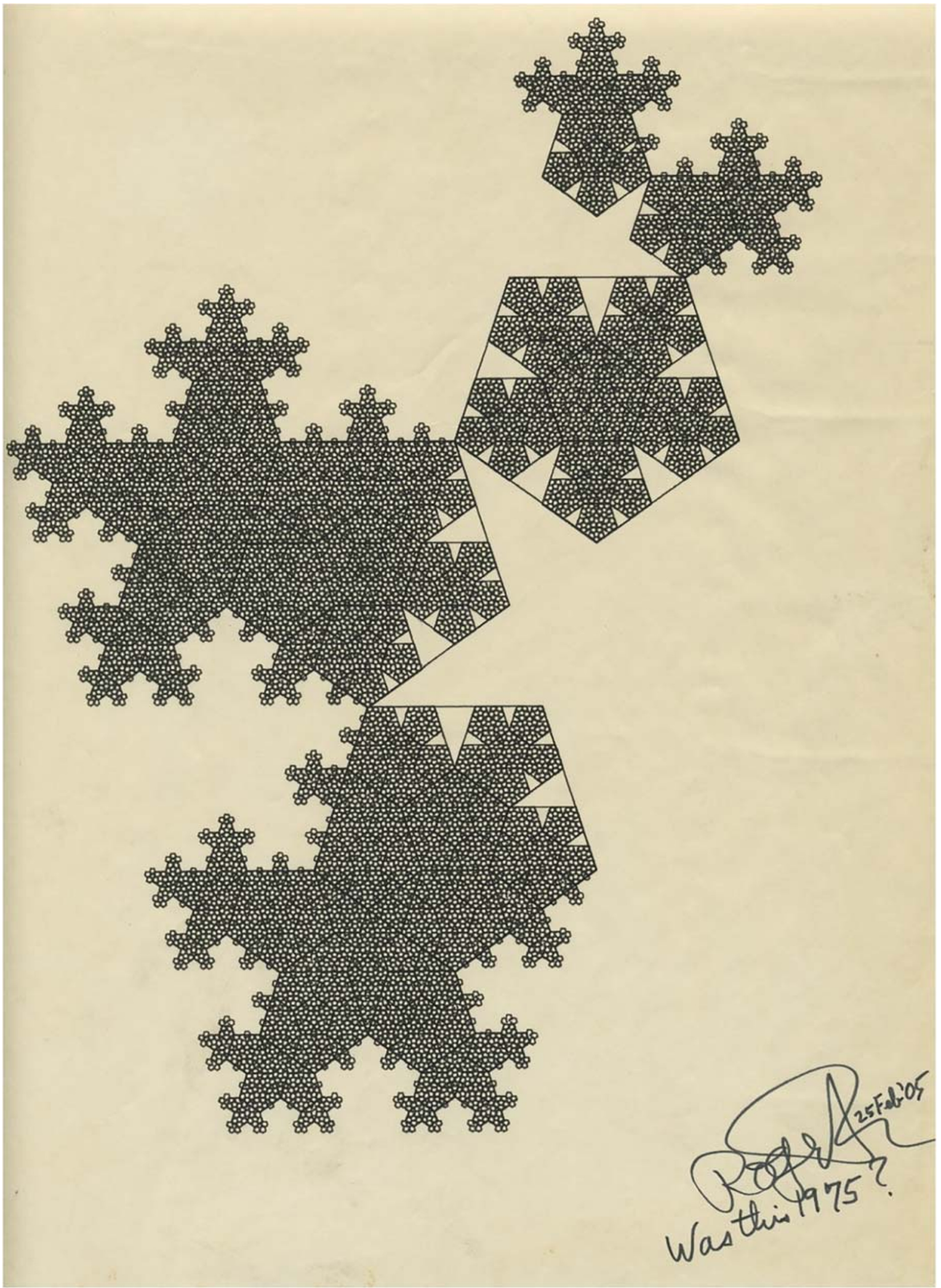

Fig. 3 Computer-automated drawing of "Pentagonal snow crystal" by Robert H. Mackay in 1975. This was originally hand-drawn by Roger Penrose, and he autographed it in 2005 (courtesy of Robert H. Mackay) 


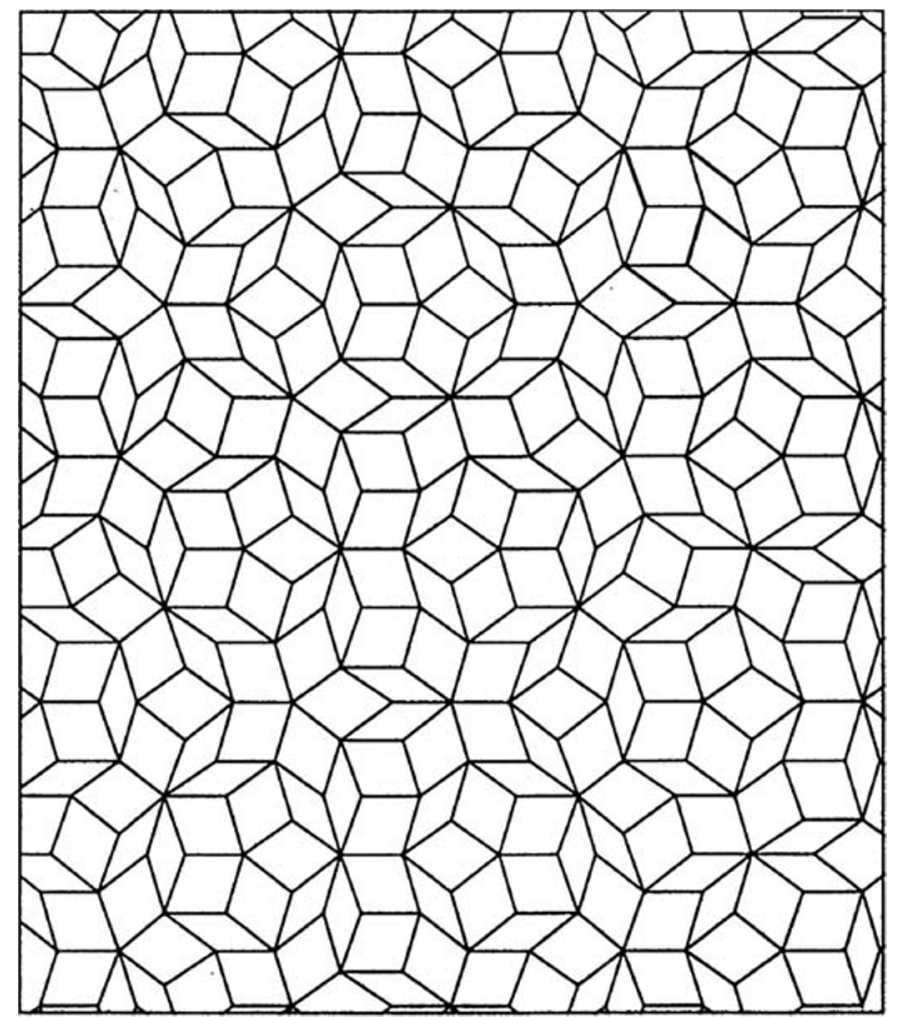

Fig. 4 The best known Penrose pattern from [6]

\section{Heroes and views}

Archimedes, Galileo, the mathematician Bernhard Riemann, and Newton are among Penrose's heroes. In his youth, Galileo was his principal source of inspiration for Galileo's courage of being against the prevailing thought. If some consider Roger Penrose (Fig. 5) a maverick, it is because of his work that is related to consciousness. Many of his contemporaries like to divide people in two clear-cut groups. Either you are just a computer, as if operating according to an algorithm, or you are

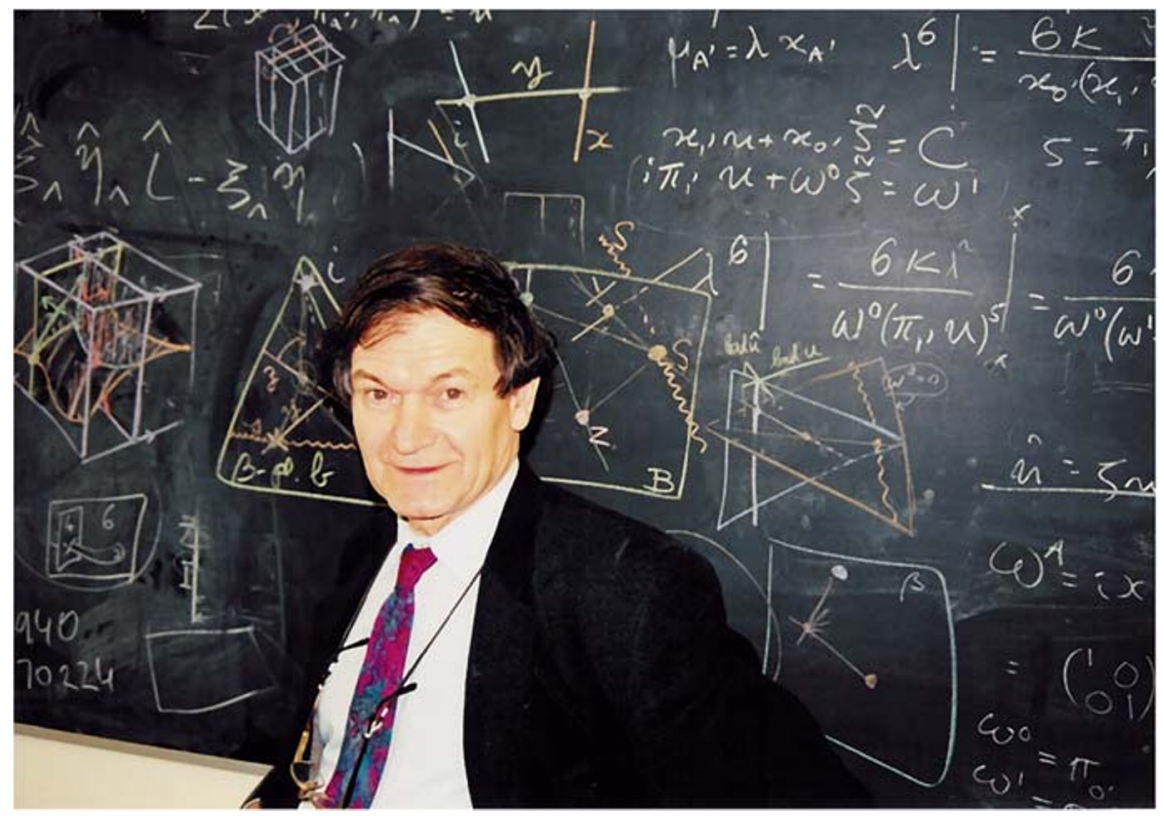

Fig. 5 Roger Penrose in 2000 in his office at Oxford University (photo by Istvan Hargittai) 
mystical, religious, and should be regarded as unscientific. Penrose emphasizes that to him conscious phenomena are real things. If there is a real phenomenon, it is part of the real world. We may not be able to explain it today, but we will be some day. The important thing is not whether or not there is yet an explanation for it; the important thing is that it be true. He knows there are people to whom quantum mechanics remains inexplicable and they interpret this by declaring quantum mechanics to be part of divine reality. Penrose realizes that there is fuzziness in the boundaries. He is not religious and it means that he does not believe any religious doctrine. However, he does not think there is nothing more than what is described by a purely reductionist view of the world. We are still learning, and even the term reductionist is not defined unambiguously. Sometimes it is meant to be scientific, which is alright. However, it is insufficient if it means that one can explain the behavior of large things in terms of the behavior of small things. Such an approach only manifests our insufficient understanding of the world. In this, we may refer to what we know and what we do not know about the black holes, in particular, when the need arises for a new physics. Some label Penrose a maverick. They dislike that he stresses our insufficient knowledge and understanding of the world. Beside the black holes, another good example is the workings of the brain. Many imagine it as if they were like the processes in a supercomputer. To Penrose, there is something more to it, and this is also why some think he is a maverick.

It is not easy to define Penrose. Like his father, he does not strictly draw a line between serious research and entertainment. He likes to understand things. He does not draw a line between his interests in mathematics and in physics. He appreciates that in British academia one is not forced to draw such lines rigorously. In the company of mathematicians, he is thought to be a physicist and in the company of physicists he is thought to be a mathematician. He does not care, but he is

\footnotetext{
${ }^{2}$ There may have been a reason for Penrose to stress that he was no businessman, because, maybe, he was, even if only to a very small extent. Many years ago there was a lawsuit because of an unauthorized use of the Penrose pattern on a toilet paper. A company, called Pentaplex, had been operating, making things based on Penrose's designs and another company encroached on it without agreement. Not much has transpired about lawsuit, because there was an out-of-court settlement. The Penrose pattern still appeared on the toilet paper following the settlement, but, by then, it did so with an agreement. The issue was not whether the pattern on the toilet paper was produced by some mathematical formula, which is public property, as mathematics is public property. The question was whether a certain pattern had been directly copied. This is an important distinction. A piece of mathematics could not be judged as being private property or public property, because it could only be judged as public property.
}

certain that he is not a businessman. ${ }^{2}$ More characteristic is that in his semi-popular books he deals with universal questions; his area of interest and expertise is the Universe itself. Some of his books, even those not too easy to read, are bestsellers. However, the number of books sold does not necessarily express the number of books read. To many, his books represent a higher level of general education. To others, keeping his books on the shelve is a status symbol.

\section{Compliance with ethical standards}

Conflict of interest The authors declare that they have no conflict of interest.

\section{References}

1. Penrose R (2016) Fashion, Faith, and Fantasy in the New Physics of the Universe. Princeton University Press, Princeton, New Jersey

2. Penrose R (2010) Cycles of Time: An Extraordinary View of the Universe. The Bodley Head, London

3. Penrose R (2007) The Road to Reality: A Complete Guide to the Laws of the Universe. Vintage, London

4. Penrose R (1994) Shadows of the Mind: A Search for the Missing Science of Consciousness. Oxford University Press, Oxford

5. Penrose R (1989) The Emperor's New Mind. Oxford University Press, Oxford

6. Hargittai B, Hargittai I (2005) Chapter 3, "Roger Penrose,”. Candid Science V: Conversations with Famous Scientists. Imperial College Press, London, pp 36-55

7. Hargittai B, Hargittai I, Hargittai M (2000-2006) Candid Science Volumes I-VI. Imperial College Press, London

8. Penrose R (1974) The role of aesthetics in pure and applied mathematical research. Bulletin of the Institute of Mathematics and Its Applications 10:266-271

9. Gardner M (1977) Extraordinary nonperiodic tiling that enriches the theory of tiles. Sci Am 236:110-121

10. Mackay AL (1982) Crystallography and the Penrose Pattern. Physica 114A:609-613

11. Shechtman D, Blech I, Gratias D, Cahn JW (1984) Metallic phase with long range orientational order and no translational symmetry. Phys Rev Lett 53:1951-1953

12. Hargittai B, Hargittai I (2012) Quasicrystal discovery - from NBS/ NIST to Stockholm. Struct Chem 23:301-306

13. Levine D, Steinhardt PJ (1984) Quasicrystals: a new class of ordered structures. Phys Rev Lett 53:2477-2480

14. Caspar M (ed) (1940) Johannes Kepler Gesammelte Werke, vol VI. C.H. Beck Verlagsbuchhandlung, München

15. Kepler J (1966) The English translation from the Latin original of De nive sexangula: The Six-cornered Snowflake. Clarendon Press, Oxford

16. Hargittai I (2011) Chapter 8, Stubbornness - "Impossible" Matter. Drive and Curiosity: What fuels the passion for science. Prometheus Books, Amherst, New York, pp 155-172

Publisher's note Springer Nature remains neutral with regard to jurisdictional claims in published maps and institutional affiliations. 